\title{
Concurrent measurement of smooth muscle shortening, lumen narrowing and flow to acetylcholine in large and small porcine bronchi
}

\author{
H.W. Mitchell, R. Cvetkovski, M.P. Sparrow, P.R. Gray, P.K. McFawn
}

Concurrent measurement of smooth muscle shortening, lumen narrowing and flow to acetylcholine in large and small porcine bronchi. H.W. Mitchell, R. Cvetkovski, M.P. Sparrow, P.R. Gray, P.K. McFawn. (OERS Journals Ltd 1998.

ABSTRACT Models of airway function indicate that responsiveness (flow reduction) to bronchoconstrictor provocation depends on airway smooth muscle shortening and airway wall morphology. The contribution of these factors to the responsiveness of central and peripheral bronchi was assessed.

Lumen flow was recorded in porcine perfused small ( $2 \mathrm{~min}$ i.d.) and large bronchial segments $(6 \mathrm{~mm}$ i.d.). Lumen diameter was recorded in the same airways after inserting an endoscope. Smooth muscle shortening, relative wall area (WAr), smooth muscle and cartilage thickness and mucosal folds were measured morphometrically. The effect of acetylcholine $\left(\mathrm{ACh} ; \mathbf{1 0}^{-6}-10^{-1} \mathrm{M}\right)$ on functional measurements was determined by curve fitting.

Maximum muscle shortening was $30 \%$ in small and $19 \%$ in large bronchi $(p<0.01)$ and lumen narrowing was $49 \%$ and $39 \%$, respectively. High doses of ACh stopped flow in small bronchi, but produced a plateau in large bronchi. Small airways were 250-times more sensitive to ACh than large airways, for all measurements. Smooth muscle and cartilage thickness and numbers of mucosal folds were greater in large than in small bronchi (pð0.01). Lumen narrowing and flow reduction were greater than predicted on the basis of muscle shortening and $W A \mathbf{r}(\mathbf{p}<0.05)$.

The structure of airways from the two groups was qualitatively similar, but responses were markedly different. Greater narrowing and flow responses of small bronchi were directly associated with smooth muscle responsiveness in situ. The results suggest that in vivo changes in airway wall shape or dimensions, or luminal secretions, exert a significant effect on airway flow, particularly in the small airways. Eur Respir J 1998; 12: 1053-1061.
Dept of Physiology, University of Western Australia, Nedlands, Australia.

Correspondence: H.W. Mitchell

Dept of Physiology

University of Western Australia

Nedlands

Perth 6907

Australia

Fax: 61893801025

Keywords: Airway narrowing asthma

bronchial responsiveness

smooth muscle shortening

Received: July 71997

Accepted after revision June 281998

Supported by the National Health and Medical Research Council of Australia. P.R. Gray was supported by an Allen \& Hanburys Respiratory Research Fellowship.
Inhaling contractile drugs, such as histamine or methacholine, narrows the airways and reduces maximal and submaximal expiratory flows measured at the mouth. Airway narrowing is produced by shortening of the airway smooth muscle, which depends on the agonist concentration at smooth muscle receptors and the intrinsic physiological properties of the smooth muscle. Simulated ideal airways indicate that airway wall thickness [1-3], mucosal folding [4] and airway secretions [5] could also affect flow or resistance by altering airway narrowing or lumen flow profiles. Alterations to some of these factors may contribute to the increased sensitivity and/or maximum response to bronchconstrictor stimuli seen in asthma [1-3]. Despite the importance of airway smooth muscle to bronchial responsiveness, the effect of muscle shortening on lumen narrowing and flow is yet to be described in an airway.

There is variability in airway narrowing amongst different airways. Morphological studies in lung or lung explants [6-10] show a wide variation in sensitivity and maximal response among different airways. Some physiological studies confirm heterogeneity in airway responsiveness in vivo [11-14]. Small (peripheral) fluid filled bronchi in vitro are more sensitive and have a greater maximum response to provocation than large (central) bronchi [15-17]. However, the range in responsiveness recorded in whole airways is not present in isolated smooth muscle cells $[15,17]$. It is, therefore, unclear whether the different physiological responses recorded in whole airways are due to variations in contraction of the smooth muscle in situ or to differences in the morphology of the airway wall.

In this study the functional narrowing behaviour of individual central and peripheral airways was examined in vitro. The first objective was to define the shortening of airway smooth muscle within the airway wall, over the complete dose-response curve to acetylcholine (ACh) and to determine whether variability in flow responses of isolated intact airways from different parts of the lung was due to variations in smooth muscle shortening. A second objective was to test the suppositions of the geometric model of airway function [1-3] in different airways and specifically, whether differences in airway narrowing and flow are explained by differences in airway wall thickness, as well as in muscle shortening. The numbers of mucosal 
folds and smooth muscle and cartilage thickness were also compared in large and small bronchi. Different functional and morphological variables were documented in the same airway and relationships between some of these variables were compared by curve fitting.

\section{Materials and methods}

\section{Preparation of bronchial segments}

Lungs were obtained from 26 pigs $(20-30 \mathrm{~kg})$. A $4 \mathrm{~cm}$ length of central (large, $6 \mathrm{~mm}$ i.d.) or peripheral (small, 2 $\mathrm{mm}$ i.d.) stem bronchus from a lower lobe was carefully dissected free of parenchyma and the side branches were tightly ligated close to the main airway trunk. Segments were mounted horizontally in a heated organ chamber containing Krebs solution $\left(37^{\circ} \mathrm{C}, 95 \% \quad \mathrm{O}_{2} / 5 \% \mathrm{CO}_{2}\right)$. The segment lumen was perfused with Krebs solution via a reservoir (set to $5 \mathrm{cmH}_{2} \mathrm{O}$ ) $[17,18]$ (fig. 1). (Note that the endoscope was not present in the airway lumen when flow was recorded; see below.)

\section{Flow}

Flow was measured by collecting perfusate from the bath outlet. With small segments, the lumen was perfused continuously and flow was monitored on alternate minutes. Large bronchi were not continuously perfused because of the large volumes required. Instead, flow was measured for $15 \mathrm{~s}$ at one-minute intervals by operating a clamp on the inlet tube. The system had a threshold detection limit of $<0.5 \mathrm{~mL} \cdot \mathrm{min}^{-1}$ flow.

Inlet and outlet tubes were kept longer than $10 \mathrm{~cm}$ to minimize flow profile distortion. Flow through the apparatus was Poiseuillian (i.e. proportional to diameter ${ }^{4}$ ). As the measured flow in the total system is dependent on both the apparatus and bronchial resistances, changes in measured flow underestimate the change in bronchial resistance produced as a result of bronchoconstriction [19]. Therefore, measured flows were corrected for apparatus resistance by subtracting this from the total system resistance to give a measure of the change in bronchial flow which would have occurred in the absence of apparatus resistance. Apparatus resistance (upstream and downstream, fig. 1) was determined by replacing the bronchial segment with a large diameter plastic tube as described previously [19].

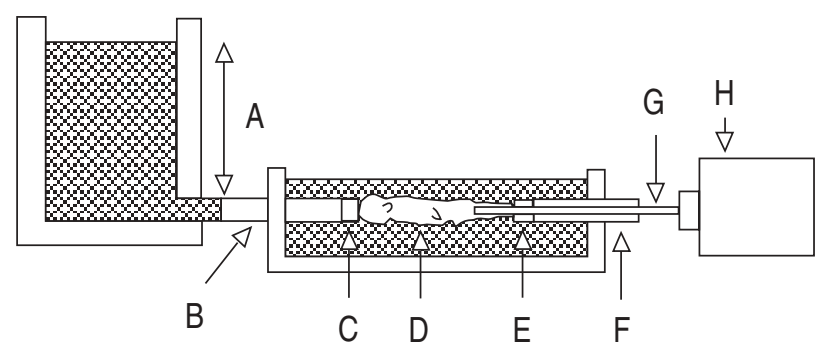

Fig. 1. - Experimental apparatus for measuring lumen flow and diameter. A: reservoir filled with Krebs solution, and pressure head; B: inlet tubing; C: proximal cannula; D: bronchial segment; E: distal cannula; F: outlet tubing; G: endoscope; $\mathrm{H}$ : video camera. The endoscope was removed for measuring flow. Contracted bronchi were fixed whilst connected to their mountings for subsequent histological and morphometric analysis.
Bronchial resistance $(R \mathrm{br})$ was

$$
R \mathrm{br}=\left(\frac{P}{F \text { tot }}\right)-R \text { ap }
$$

and bronchial flow was

$$
\frac{P}{R \mathrm{br}}
$$

where $P$ is driving pressure $\left(5 \mathrm{cmH}_{2} \mathrm{O}\right), F$ tot is total flow, and $R$ ap is apparatus resistance.

\section{Bronchial pressure drop}

During flow there is a pressure drop along the bronchial segment, resulting in a graded change in transmural pressure. Upstream and downstream resistances were calculated to determine the transmural pressures in the proximal and distal ends of the segment. Upstream resistance was calculated from flow through the upstream apparatus and the pressure head. Downstream resistance was calculated by subtracting upstream resistance from total apparatus resistance. Pressures were then calculated from the product of resistances and the measured flow.

\section{Narrowing}

Lumen diameter was recorded using a fibreoptic endoscope [20] (fig. 1). Measurements were made $1 \mathrm{~cm}$ from the distal end of each segment. To aid visualization of the region of interest the lumen was painted with a dye ring, which was also used to locate the region of the bronchus to be studied morphometrically. The endoscope was inserted to within $1 \mathrm{~cm}$, of the dye ring, at which distance there was no lateral image distortion. The image was subsequently analysed using VideoPro image analysis software (Leading Edge, Adelaide, South Australia).

Bronchi were not perfused when recording lumen diameter. To maintain the transmural pressure, the distal end of the bronchus was sealed and the pressure head was reduced to $2.5 \mathrm{cmH}_{2} \mathrm{O}$. Lumen recordings were made before and after adding ACh. The lumen diameter was calculated using the perimeter of the dyed region, assuming circularity. The mucosal fold interstices were not visible and so were not included in the measurement of lumen diameter. Circularity was investigated in contracted and relaxed airways by comparing the lumen area measured with the image analysis software, with the area of a circle defined from the lumen perimeter at the dye ring [20]. The plot of the measured versus calculated areas will have a unitary slope if the lumen is circular. In relaxed small and large bronchi the slope was $0.84 \pm 0.02$ and $0.87 \pm 0.02$, respectively, and $0.88 \pm 0.01$ and $0.86 \pm 0.01$ in contracted small and large bronchi.

\section{Muscle contraction}

Bronchi were fixed whilst mounted in the perfusion chamber, at $2.5 \mathrm{cmH}_{2} \mathrm{O}$ pressure and not perfused, by adding $10 \%$ formalin solution to the bath for $1 \mathrm{~h}$ at $37^{\circ} \mathrm{C}$. Segments remained mounted overnight in the fixative at room 
temperature and were then removed from the bath and placed in $10 \%$ formalin for a further $24 \mathrm{~h}$. In several experiments the lumen was monitored during the first hour of fixation to ensure that the diameter did not change.

Morphometric measurements were made on nine frozen sections from a $1 \mathrm{~mm}$ length of the segment in the region imaged endoscopically. Measurements included the lengths of the epithelium $(\mathrm{Li})$ and of the outer margin of the smooth muscle $\left(L_{e}\right)$ and the area of the bronchial lumen $(A \mathrm{i})$. The cross-sectional area from the luminal margin of the epithelium to $L e$ was the inner wall area (WAi). The relative wall area (WAr) was the ratio of the WAi to $W A$ i plus $A \mathrm{i}$, when the lumen is circular. The area associated with the hydraulic diameter (i.e. the lumen area to the tips of the mucosal folds) was subtracted from the total lumen area, which included the interstices. The amount of circumferential smooth muscle shortening produced by $\mathrm{ACh}$ in each bronchial segment was estimated by the method of JaMES et al. [21]. Muscle shortening produced by ACh was corrected for the small amount of shortening ( $2.7 \%$ in small bronchi and $<1 \%$ in large bronchi) present in separate control bronchi not exposed to ACh. The average thickness of the smooth muscle and cartilage layers was determined in a separate experiment (four large and four small relaxed bronchi) from their areas, assuming annularity.

\section{Protocols}

One hour after mounting the segment, a submaximal dose of ACh was added to the bath to give a standard contraction history. Upon recovery, each bronchial segment was stimulated with a dose of ACh selected to produce a response between threshold and supramaximum (10-6-10-1 $\mathrm{M})$. The effect of the selected ACh dose on lumen flow was first determined then the endoscope was inserted 1-2 $\mathrm{h}$ after washout and recovery and the effect of the same dose of ACh was determined on lumen diameter. Once the diameter of the lumen had been measured, the bronchus was fixed while still contracted, for subsequent morphometry and estimation of muscle shortening.

Curves of best fit were applied to pooled data for each airway group to establish both a dose-response relationship and some of the dynamic relationships between muscle shortening and lumen flow and diameter. These relationships were then compared to predicted relationships for ideal airways where flow is Poiseuillian, and where lumen narrowing is fully defined by muscle shortening and the $W A r$. The WAr used in the predicted relationships were the mean values obtained morphometrically from the large or small bronchi employed in the study.

\section{Analysis of data}

Dose-response curves were plotted for pooled values of flow, lumen diameter (recorded endoscopically) and muscle shortening (estimated morphometrically) from large or small airways. Data were fitted to a hyperbolic curve (i.e. the Michaelis-Menton expression):

$$
\text { Response }=\frac{a x^{2}}{b^{2}+x^{2}}
$$

The experimental relationship between lumen diameter (fractional diameter $\left(D_{\mathrm{f}}\right)$, diameter with $\mathrm{ACh} /$ resting diameter) and flow (fractional flow $\left(F_{\mathrm{f}}\right)$, adjusted flow with $\mathrm{ACh} /$ resting adjusted flow) was plotted using:

$$
F_{\mathrm{f}}=D_{\mathrm{f}}^{a}
$$

where $a$ is the best-fit power function. For plotting a predicted relationship for Poiseuillian flow, $a$ is 4 .

The experimental relationship between muscle length (fractional muscle length $\left(M L_{\mathrm{f}}\right), 100$ minus \%muscle shortening/ 100) and lumen diameter was plotted using the equation of Moreno et al. [1]:

$$
D_{\mathrm{f}}=\frac{Ð \overline{\left(M L_{\mathrm{f}}^{2}-b\right)}}{Ð \overline{(1-b)}}
$$

where $b$ is the best-fit value for WAr. For plotting a predicted relationship, $b$ is the mean value of WAr which was determined morphometrically on the bronchi used.

The experimental relationship between muscle length and lumen flow was plotted using:

$$
F_{\mathrm{f}}=\left(\frac{Ð \overline{\left(M L_{\mathrm{f}}-b\right)}}{Ð \overline{(1-b)}}\right)
$$

where $b$ is the best-fit value for WAr. For plotting a predicted relationship, $b$ is the the mean value of WAr which was determined morphometrically on the bronchi used.

\section{Statistics}

Coefficients of determination $\left(\mathrm{r}^{2}\right)$ for the best-fit curves applied to experimentally derived data were calculated by the method of least squares. The Chi-squared test was used to determine whether experimental data points in an airway group were significantly different from predicted curves (see above). Maximum changes in flow, muscle shortening and lumen diameter were calculated from the mean of values recorded for the highest five doses of $\mathrm{ACh}$ (i.e. at the plateau of the dose-response curves in figs. 2-4) in

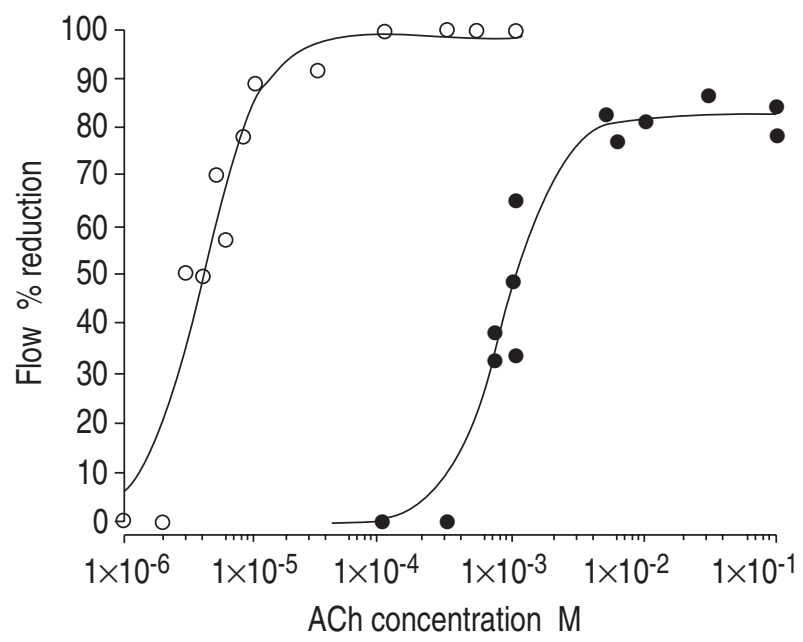

Fig. 2. - Effect of acetylcholine (ACh) on lumen flow in small (O; 2 $\mathrm{mm}$ i.d.) and large (•; $6 \mathrm{~mm}$ i.d.) bronchi. Each point is from one bronchial segment. Best-fit curve from equation 3. $\mathrm{r}^{2}=0.9426(\mathrm{n}=13)$ in small bronchi and $\mathrm{r}^{2}=0.9450(\mathrm{n}=13)$ in large bronchi. 




Fig. 3. - Effect of acetylcholine (ACh) on lumen diameter in small ( $\bigcirc$; $2 \mathrm{~mm}$ i.d.) and large ( $6 \mathrm{~mm}$ i.d.) bronchi. Lumen diameter was recorded with an endoscope. Each point is from one bronchial segment. Best-fit curve from equation 3. $r^{2}=0.9448(n=13)$ in small bronchial and $\mathrm{r}^{2}=0.8233(\mathrm{n}=13)$ in large bronchi.

each group. A Student's t-test was used to compare the mean maximum effects in small- and large-bore airways. A $p<0.05$ was regarded as significant. Data are means \pm SEM. The number of bronchi and animals was 13 except where specified differently.

\section{Results}

Dose-response curves from pooled data in each airway size closely fitted a hyperbolic function (equation 3 ) with coefficients of determination $\left(\mathrm{r}^{2}\right)$ between $0.7327-0.9448$ for the different measurements (figs. 2-4). There was a marked difference in the sensitivity of the two groups of airways to ACh, with an approximate 250-fold decrease in the median effective concentration (EC50) in small bronchi compared with large bronchi for flow, lumen narrowing and muscle shortening (figs. 2-4, table 1).

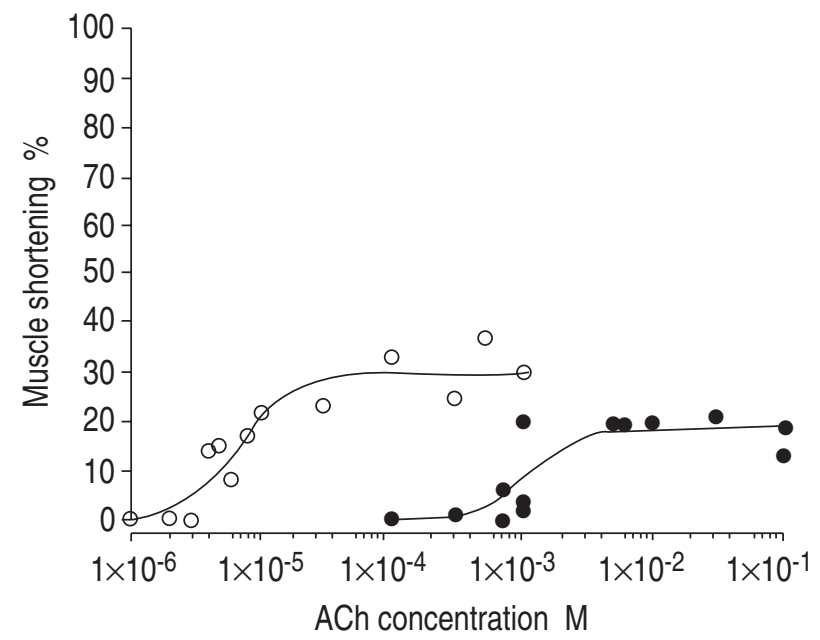

Fig. 4. - Effect of acetylcholine (ACh) on muscle shortening in small ( $0 ; 2 \mathrm{~mm}$ i.d.) and large ( $\bullet 6 \mathrm{~mm}$ i.d.) bronchi. Muscle shortening was estimated morphometrically. Each point is from one bronchial segment. Best-fit curve from equation 3. $r^{2}=0.8785(n=13)$ in small bronchi and $\mathrm{r}^{2}=0.7327(\mathrm{n}=13)$ in large bronchi.
Table 1. - Responsiveness of small and large bronchi

\begin{tabular}{|c|c|c|c|c|}
\hline & \multicolumn{2}{|c|}{ Small bronchi } & \multicolumn{2}{|c|}{ Large bronchi } \\
\hline & $\overline{\mathrm{pD}_{2}}$ & Emax & $\overline{\mathrm{pD}_{2}}$ & Emax \\
\hline Lumen flow & 5.40 & 100 & 3.08 & $83 * * *$ \\
\hline Lumen diameter & 5.16 & 48 & 3.01 & 39 \\
\hline Muscle shortening & 5.17 & 30 & 2.93 & $19 * *$ \\
\hline
\end{tabular}

$\mathrm{pD}_{2}$ : sensitivity (-log median effective concentration (EC50)) to acetylcholine (ACh); Emax: maximum effect (\% change) of ACh on small $(n=13)$ and large $(n=13)$ bronchial segments. Flow, narrowing and muscle shortening was measured in each bronchial segment. Each bronchus was stimulated with a single dose of ACh. Responses in all bronchi in a group were then pooled and best-fit curves were drawn. Parameters of responsiveness were calculated from the equation of best-fit. **: p<0.01; ***: $\mathrm{p}<0.001$, between small and large airways (mean of highest five doses of $\mathrm{ACh}$ ).

Flow

Small and large bronchi had resting flows of $0.184 \pm$ 0.015 and $5.5 \pm 0.7 \mathrm{~L} \cdot \mathrm{min}^{-1}$, respectively. Maximum ACh doses stopped flow in small bronchi and reduced it to a plateau at $83 \%$ in large bronchi (fig. 2).

\section{Bronchial pressure drop}

In relaxed bronchi, the transmural pressure at the proximal to distal ends ranged $3.3 \pm 0-1.6 \pm 0 \mathrm{cmH}_{2} \mathrm{O}$ for small bronchi and $4.8 \pm 0-3.7 \pm 0.1 \mathrm{cmH}_{2} \mathrm{O}$ for large bronchi, respectively $(n=13)$. After contraction, the proximal transmural pressure increased whilst the distal decreased depending on the extent of stimulation. For maximally stimulated small bronchi the proximal to distal pressure range became $4.9 \pm$ $0.1-0.1 \pm 0.1 \mathrm{cmH}_{2} \mathrm{O}$, while in large bronchi it became $4.9 \pm$ $0-1.9 \pm 0 \mathrm{cmH}_{2} \mathrm{O}(\mathrm{n}=5)$.

\section{Lumen narrowing}

Endoscopically measured lumen diameters averaged $2.0 \pm 0.1 \mathrm{~mm}$ in relaxed small bronchi $(1.6-2.6 \mathrm{~mm})$ and $5.7 \pm 0.3 \mathrm{~mm}$ in large bronchi $(5.1-8.3 \mathrm{~mm})$. Morphometric estimates of lumen diameter agreed closely, i.e. 1.9 \pm 0.1 $\mathrm{mm}$ in small and $5.5 \pm 0.2 \mathrm{~mm}$ in large bronchi. Lumen narrowing (fig. 3) plateaued at high ACh doses. Maximum $\mathrm{ACh}$ doses reduced the lumen diameter by $48 \pm 3 \%$ and $39 \pm 5 \%$ in small and large bronchi, but never completely closed the bronchial lumen (fig. 3). A maximally stimulated small bronchial lumen is shown in figure 5c. No occlusion was visible either endoscopically or histologically in other parts of the airway lumen, although flocculant mucus was present in the lumen of some bronchi after ACh stimulation (fig. 5c).

\section{Muscle shortening}

Muscle shortening plateaued at high ACh doses (fig. 4). Maximum muscle shortening was $30 \pm 3 \%$ in small and $19 \pm 2 \%$ in large bronchi $(\mathrm{p}<0.01)$. Sections of small bronchi were studied with a $\times 4$ objective and the coefficient of variation $(\mathrm{CV})$ for repeat measurements of muscle shortening was $1.4 \%$ ( $n=5$ measurements in one bronchus). A $\times 1$ objective was used for large bronchi and the CV was $9.1 \%$. In contracted bronchi there was partial separation between the smooth muscle and the cartilage (fig. 5). 

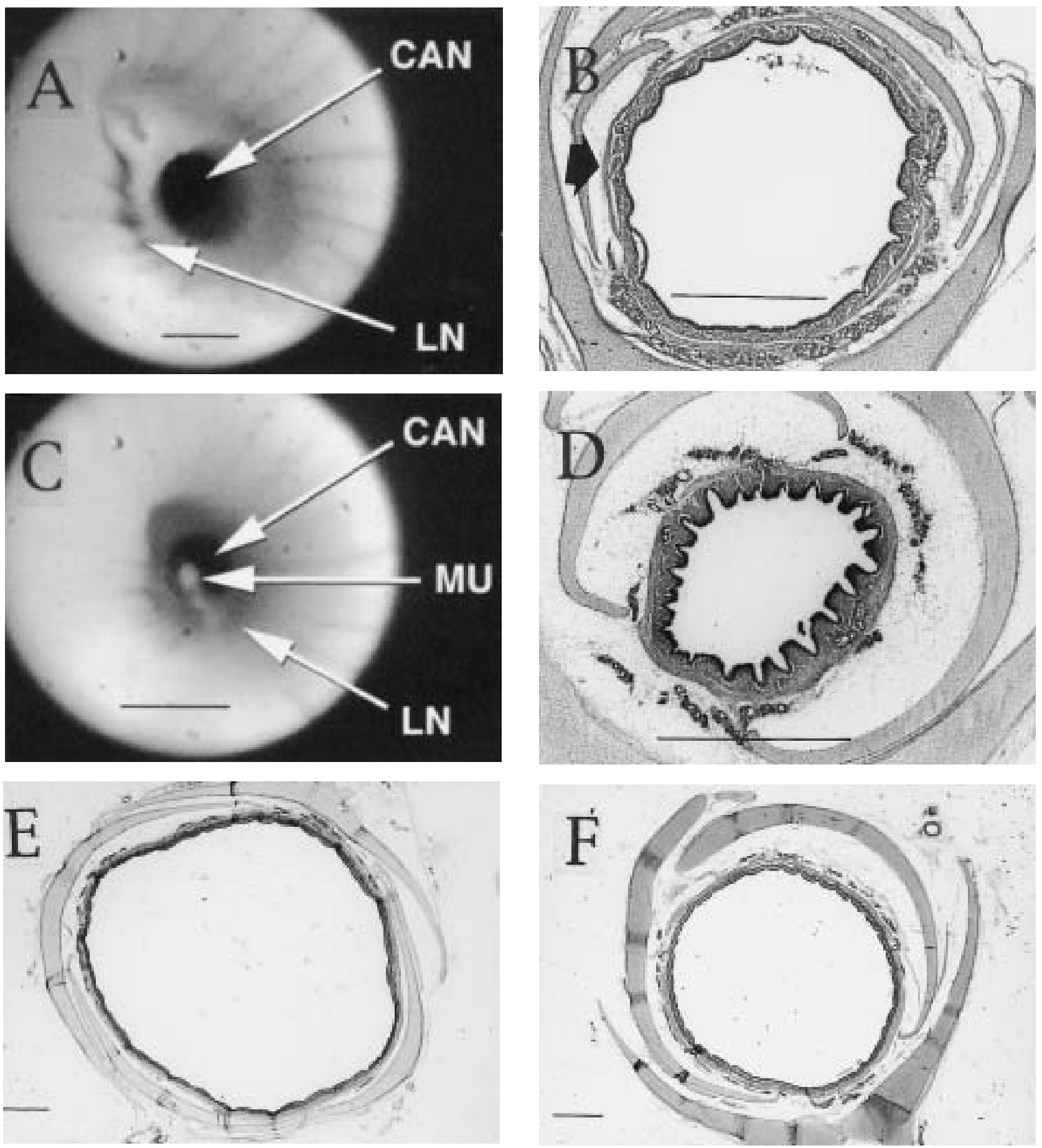

Fig. 5. - Lumen and airway wall of bronchial segments before and after exposure to high doses of acetylcholine (ACh). A) Image of the lumen of a relaxed small-bore bronchus taken through an endoscope. Note the sidebranch, line of dye applied to the mucosa (LN) and the furthest end of the bronchial segment near its cannula (CAN). B) Histological section of a different relaxed small-bore bronchus stained with haemotoxylin and eosin. The outer margin of the smooth muscle is identified by an arrow. C) Endoscopic image of the same bronchus in A) but during exposure to $10^{-4} \mathrm{M} \mathrm{ACh}$. In a previous run, this dose of ACh caused flow to fall to zero. The furthest end of the bronchus, near its cannula (CAN), was still visible along the lumen. Some flocculant mucus and a small mucus mass (MU) were present in the lumen. D) The same bronchus as in C) after fixation during ACh exposure. The inner airway wall was partially and reversibly separated from the cartilage during ACh exposure. E, F) Relaxed and contracted (10-2 $\mathrm{M} \mathrm{ACh})$ largebore bronchial segments. (Internal scale bars $=1 \mathrm{~mm}$.)

\section{Airway morphology}

In small bronchi there were $20 \pm 1$ mucosal folds $\cdot a i r w a y-1$ and in large bronchi there were $36 \pm 3$ folds airway ${ }^{-1}(\mathrm{p}<$ 0.001 , fig. 5). Mucosal fold numbers did not correlate with muscle shortening in either group. However, the areas of the fold interstices were positively correlated with muscle shortening $(\mathrm{p}<0.01-0.05)$. In small airways the area of the interstices ranged from $2 \%$ of the lumen area $(A \mathrm{i})$ in relaxed bronchi to $10 \%$ in fully contracted bronchi. In large airways the areas ranged $0.5-2 \%$ of the lumen area. Relative wall areas $(W A r)$ were $0.185 \pm 0.009$ in small and 

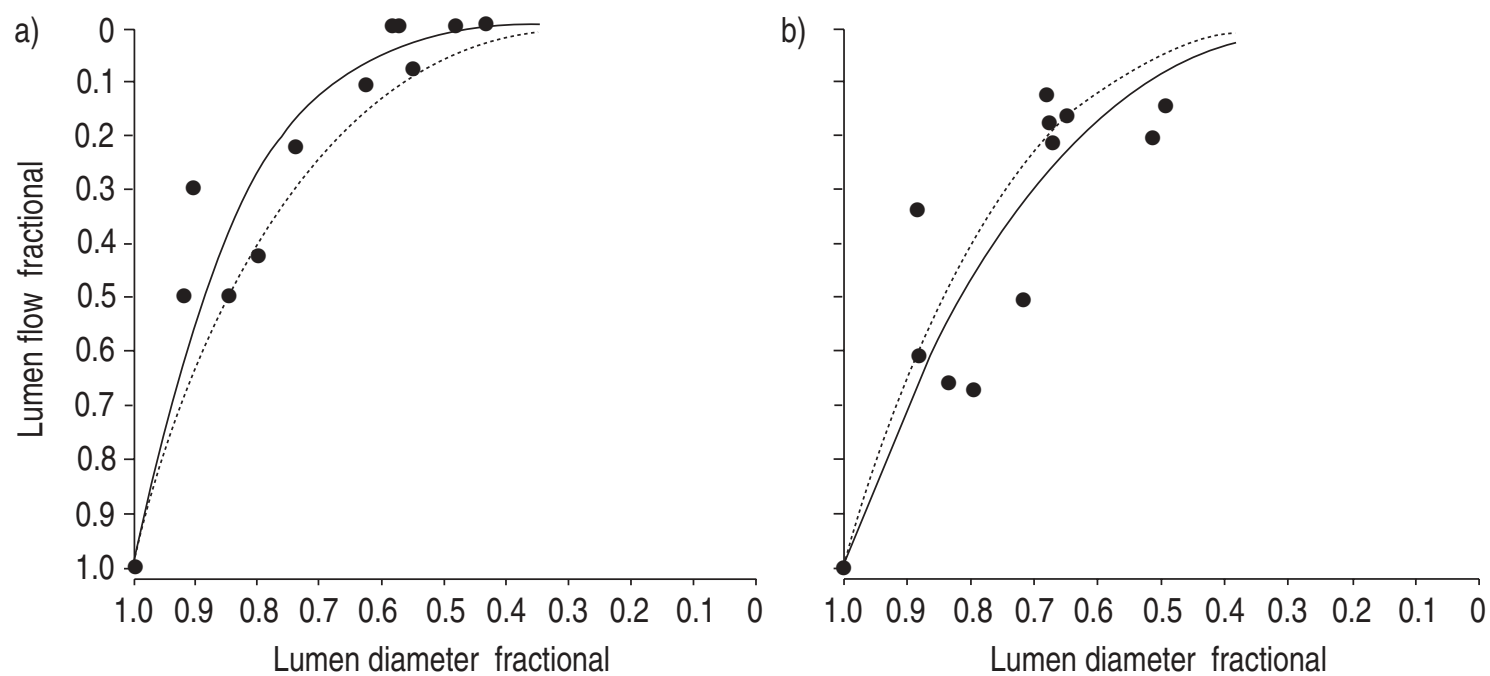

Fig. 6. - Relationship between lumen diameter and lumen flow in a) small and b) large bronchi. The points are from the same bronchial segments shown in figures 2-4. Data are expressed as fractions (i.e. value with acetylcholine/resting value). The best-fit curve for the experimental data ( $\longrightarrow$ ), using equation 4 is shown, $r^{2}=0.9140(n=13)$ in small bronchi and $r^{2}=0.8010(n=13)$ in large bronchi. Curves for a predicted fourth-power relationship are also shown $(\cdots \cdots \cdot)$. In small bronchi (but not large bronchi) the experimental data were significantly different ( $\mathrm{p}<0.05$, Chi-squared test) from the predicted curve.

$0.125 \pm 0.006$ in large airways $(\mathrm{p}<0.001)$. Average smooth muscle thickness was $0.026 \pm 0.002 \mathrm{~mm}$ and $0.082 \pm 0.006$ $\mathrm{mm}$ in small and large bronchi respectively $(\mathrm{p}<0.001, \mathrm{n}=4$ separate bronchi). The average cartilage thickness was $0.469 \pm 0.046 \mathrm{~mm}$ in small and $0.757 \pm 0.034 \mathrm{~mm}$ in large bronchi $(\mathrm{p}=0.01, \mathrm{n}=4$ separate bronchi).

\section{Flow versus diameter}

In large bronchi the experimentally derived measurements of lumen flow and lumen diameter were not significantly different to a predicted relationship for Poiseuillian flow (fig. 6). In small airways, data were similar to that predicted, but with high levels of stimulation in four bronchi, flow was stopped and the relationship between diameter and flow departed from that predicted $(\mathrm{p}<0.05)$. The

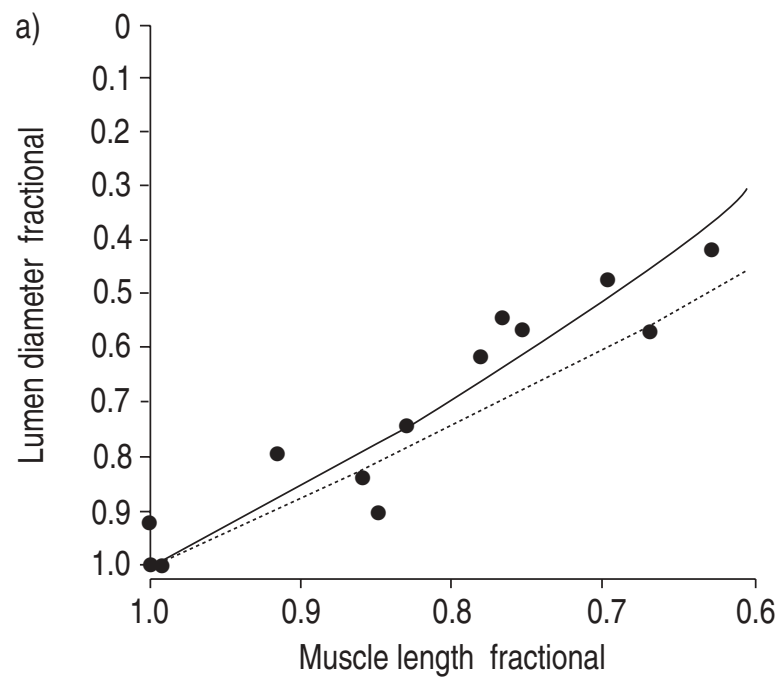

best-fit curves applied to the experimental data (equation 4) had power functions of 3.4 in large bronchi (flow $\alpha$ diameter ${ }^{3.4}$ ) and 5.7 (flow $\alpha$ diameter ${ }^{5.7}$ ) in small bronchi.

\section{Muscle shortening versus diameter}

Figure 7 plots a relationship between muscle length and lumen diameter. In small bronchi, the experimental data were similar to the relationship predicted from a geometric model (equation 5). In large bronchi, lumen narrowing was greater than predicted $(\mathrm{p}<0.05)$. The curve of best-fit applied to the experimental data (equation 5) had best-fit values of WAr of 0.289 in small and 0.403 in large bronchi, which were greater than the measured values (see above).

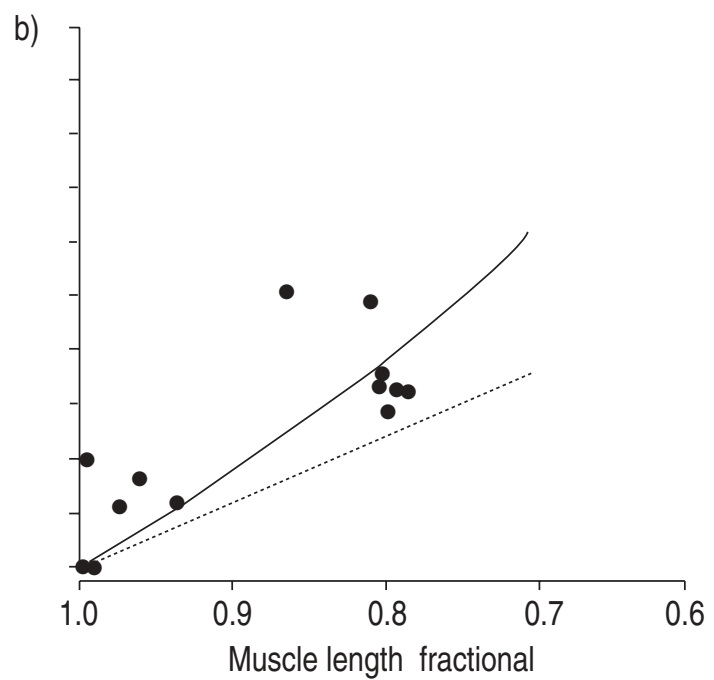

Fig. 7. - Relationship between muscle length and lumen diameter in a) small and b) large bronchi. The points are from the same bronchial segments shown in figures 2-4. Data are expressed as fractions (i.e. value with acetylcholine/resting value). The best-fit curve for the experimental data ( - ), using equation 5 is shown, $\mathrm{r}^{2}=0.8766(\mathrm{n}=13)$ in small bronchi and $\mathrm{r}^{2}=0.5810(\mathrm{n}=13)$ in large bronchi. The predicted relationship $(\cdots \cdots \cdot)$, using the actual mean inner wall areas of the airway group (relative wall area $(W A r)=0.185$ in small and 0.125 in large bronchi) in equation 5 is shown. In small bronchi the experimental data were not significantly different from the predicted curve, but they were so in large bronchi ( $<<0.05$, Chi-squared test). 

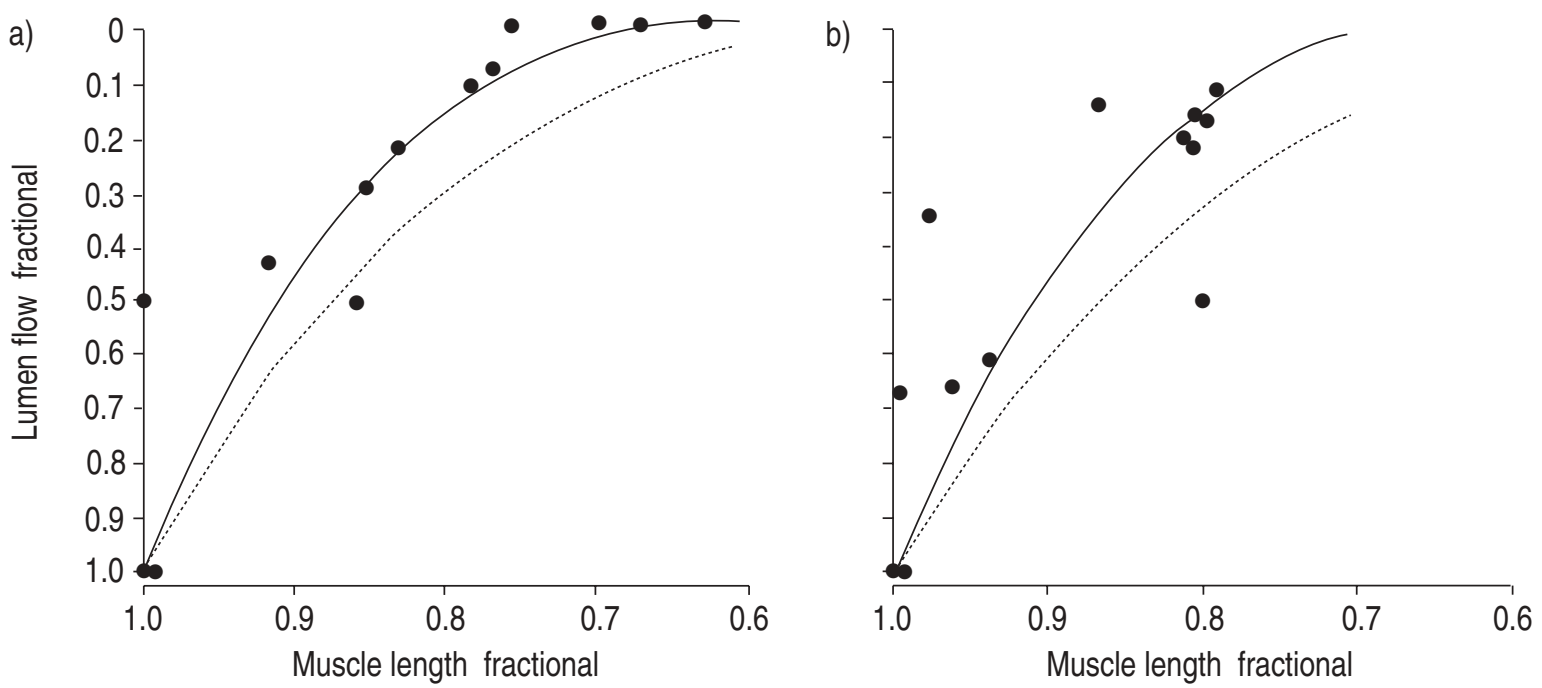

Fig. 8. - Relationship between muscle length and lumen flow in a) small and b) large bronchi. The points are from the same bronchial segments shown in figures 2-4. Data are expressed as fractions (i.e. value with acetylcholine/resting value). The best-fit curve for the experimental data ( $\longrightarrow$ ), using equation 6 is shown, $r^{2}=0.8283(n=13)$ in small bronchi and $r^{2}=0.6720(n=13)$ in large bronchi. The predicted relationship $(\cdots \cdots)$, using the actual mean inner wall areas of the airway groups (relative wall area $(W A r)=0.185$ in small and 0.125 in large bronchi) in equation 6 is shown. In both sizes of bronchi the experimental data were significantly different from the predicted curves $(\mathrm{p}<0.05$, Chi-squared test).

\section{Muscle shortening versus flow}

The relationship between muscle length and lumen flow is shown in figure 8. In large and small airway, the flow reduction caused by muscle shortening was greater than predicted from a geometric model (equation 6) $(\mathrm{p}<0.05)$. The best-fit curve applied to the data (equation 6) had best-fit values of WAr of 0.390 in small and 0.386 in large bronchi.

\section{Discussion}

In this study, complete ACh dose-response curves for muscle shortening, lumen narrowing, and lumen flow were documented in the same airway. This enabled some of the relationships which may determine responsiveness in individual airways to be defined, and potential causes for regional differences in responsiveness to be identified. Small bronchi were significantly more sensitive to ACh than large airways for all measurements. Maximum flow reduction, muscle shortening and lumen narrowing were also greater in small than in large bronchi. The greater responsiveness (flow) in small versus large bronchi could be partly explained by a greater sensitivity and maximum effect (Emax) of smooth muscle shortening. Flow was reduced more than expected, on the basis of muscle shortening and $W A$, so that small bronchi were prone to flow cessation.

Previously, the effects of agonist drugs on lung resistance were studied in whole lungs in conjunction with morphometric estimates of airway calibre in broad samples of airways [6-9]. The current protocol, in the individual live airway, was to record the effect of ACh on lumen flow then, after recovery, to insert an endoscope into the lumen of the same airway and record the effect of ACh challenge on lumen diameter. An endoscope was used so that the change in calibre could be directly established. Measurements of resting diameter (i.e. prestimulated) ob- tained using the endoscope were internally consistent with diameters estimated morphometrically from airway perimeter ( $\mathrm{Li}$; see Materials and methods). Finally, the contracted bronchi were fixed for morphometry.

Airway responses recorded in the present study could be influenced by the transmural pressure [22-24]. Muscle shortening and lumen narrowing were each recorded at $2.5 \mathrm{cmH}_{2} \mathrm{O}$ transmural pressure, without flow, and at the same location along the airway. In contrast, when flow was recorded there was a pressure drop along the airway. The $2.5 \mathrm{cmH}_{2} \mathrm{O}$ transmural pressure applied to the airway when narrowing and muscle shortening were recorded was chosen as it was a mid-range pressure at the region of flow limitation during flow $\left(1.6 \mathrm{cmH}_{2} \mathrm{O}\right.$ in relaxed small bronchi and $3.7 \mathrm{cmH}_{2} \mathrm{O}$ in relaxed large bronchi, at their distal ends). Variations in transmural pressure along segments during flow, and during ACh-induced constriction, could change airway load, and thus, complicate a comparison of an effect of ACh on flow ( $c f$. lumen narrowing and muscle shortening). However, it was previously shown that the cartilaginous airways used in these studies are less sensitive to variations in transmural pressure than resistance airways in vivo, or human airways in vitro [25]. Neither airway narrowing [20] nor flow responses $[17,26]$ are sensitive to small changes in transmural pressure $\left(\sim 5 \mathrm{cmH}_{2} \mathrm{O}\right)$. Similarly, muscle shortening is unaffected by small changes in transmural pressure along the length of perfused segments (J.C. Marriott, University of Western Australia, personal communication).

Flow, lumen narrowing and muscle shortening were dose dependent and closely fitted a hyperbolic equation in each group (which defines the kinetics of drug-receptormediated pharmacological responses). There was a 250fold greater sensitivity in muscle shortening and lumen narrowing in small than in large bronchi, which closely matched the difference in flow, as seen previously [17]. Small differences in flow profiles between large and small perfused bronchi (see below) could not account for the major difference in sensitivity. Interestingly, this sensitivity 
difference is only seen in intact airways. Isolated smooth muscle cells from similar airways do not exhibit differences in sensitivity to cholinergic agonists [15]. The threedimensional arrangement of smooth muscle in the airway wall could influence force generation and smooth muscle shortening [27], as could mural elastic loads. These could all vary with airway size or location and would be more likely to affect the responsiveness of the intact airway. A further influence could be the presence of agonist concentration gradients across the airway wall, which could differ between small and large airways [15, 17].

Another striking difference between small and large airways was in the Emax. In large airways, flow plateaued, but in small airways it stopped abruptly. The present study of small bronchi demonstrated a plateau in smooth muscle shortening, and lumen narrowing at about a 50\% reduction of diameter. This is similar to the reduction in lumen diameter in some in vivo studies [12, 28-30] and is consistent with a previous report of maximum airway narrowing in pig bronchi, recorded at zero transmural pressure [20]. Halving the lumen diameter would lead to an approximate $95 \%$ reduction in flow if flow was Poiseuillian (see below). The observation of flow cessation in small bronchi was complicated by an uncertain transmural pressure at the actual site(s) of flow limitation, so that the load on the smooth muscle during flow may have been different to the load when lumen narrowing was measured. The possibility that there may have been localized regions of airway closure during investigations of flow cannot be ruled out.

Maximum muscle shortening was also greater in small than in large bronchi, consistent with the greater Emax for flow in small than in large bronchi. Maximum muscle shortening, established from complete dose-response curves in individual bronchi, has not to the author's knowledge been previously determined. Previously reported muscle shortening in small bronchi was similar to [6] or greater than [31] that found here. The greater maximum muscle shortening in small versus large bronchi was not due to a lack of smooth muscle in large bronchi, which were three times thicker. Differences could be due to different elastic loads [6, 22, 32], for example, from the airway wall, or to intrinsic physiological properties of the smooth muscle. ОкаZаWA et al. [31] demonstrated an inverse relationship between $W A \mathrm{r}$ and muscle shortening in a sample of different sized airways in whole lung, suggesting that the airway wall may load the smooth muscle. The present airway samples were tightly grouped, but between the groups WAr and muscle shortening were directly related, suggesting that the wall area was not a key factor in smooth muscle shortening. In addition, a smaller load from mucosal folds [4] did not appear to account for greater smooth muscle shortening in the small bronchi $\left(\sim 10\right.$ folds $\cdot \mathrm{mm}$ internal perimeter $\left.{ }^{-1}\right)$ compared with large bronchi $\left(\sim 6.5\right.$ folds $\left.\cdot \mathrm{mm}^{-1}\right)$. Both large and small bronchi contained cartilage, which was almost twice as thick in the larger bronchi and could impede muscle shortening. In previous studies [33, 34], a partial and reversible separation between contracted smooth muscle and cartilage was demonstrated, which was again confirmed here (fig. 5). Since smooth muscle can partially uncouple from the cartilage, it is highly improbable that cartilage thickness per se limits muscle shortening. The extent of smooth muscle shortening might be more influenced by the compliance of the connective tissue matrix. Currently, no information is available on the elastic properties of this matrix in large or small airways.

The present findings suggest there was more flow reduction than expected from muscle shortening and WAr, in each airway group (fig. 8). This was consistent with a steeper than expected relationship between muscle shortening and lumen diameter, but which was only significant in large bronchi. The spread of experimental data in this analysis of large airways ( $c f$. small) limits comparison of a possible difference between the airway groups. Curves (experimental or predicted) were constructed from equations for a geometric model [1-3] of airway narrowing or flow (equations 5 and 6), in which the measured muscle shortening was the independent variable. Predicted relationships were constructed using the mean WAr measured morphometrically in each group. In the experimental model best-fit $W A$ r were iterated in the curve-fitting procedure and yielded $W A r$ that were greater than those measured morphometrically. Thus, the airway behaved as if the wall were thicker than actually measured and showed more narrowing and flow reduction than theoretically expected as the muscle shortened.

The reason for airway narrowing and flow being greater than expected was not established, but some possibilities can be considered. 1) The relationship between muscle shortening, flow, and narrowing is independent of variables such as the ACh concentration at the smooth muscle; the relationship between muscle shortening and lumen narrowing is also independent of load in the airway wall. 2) The choice of medium does not influence analyses of fractional flows in our study, nor should the presence of boundary layers, which are very thin. Water flow is less than air flow, but the change in flow produced by diameter changes would be similar for water and air. 3) The geometric model assumes that the airway wall is fully conserved during contraction. There is some morphometric evidence supporting this $[3,33]$, but recent data also suggest that parts of the airway, such as the outer wall area, alter during bronchoconstriction [33-35], changing airway geometry and possibly airway narrowing. 4) Real airways are not truely circular, in contrast to the ideal airway, and the lumen has irregularities due to mucosal folds and interstices. The area contained in the fold interstices is only a small proportion of the lumen area (present study, [5]). The endoscopic technique used to assess lumen narrowing did not include the area of these interstices in the measurement because the interstices are hidden from view. However, the analysis of flow versus diameter (fig. 6) was generally consistent with a model in which flow only occurs through the centre of the lumen and not through interstices. 5) Lastly, a lining of mucus could significantly reduce lumen flow, particularly in partially constricted peripheral airways by occluding the lumen or by increasing viscosity. Flocculent and sometimes viscous mucus were frequently observed endoscopically and the concentration of mucus proteins in perfusion fluid increased after ACh stimulation (unpublished observation).

In vivo, many factors potentially influence responsiveness, in addition to the physiological properties of individual airways examined in this study. These include parenchymal interdependence, parallel resistances in the bronchial tree, and compliance. The central and peripheral bronchi studied were structurally similar, in proportion to 
airway diameter. The study shows that differences in responsiveness of individual airways are, in part, caused by differences in sensitivity and the maximum effect of smooth muscle shortening. However, there was insufficient smooth muscle shortening to account for the low flows produced by acetylcholine in either large or small airways or the flow cessation in small airways (subject to the experimental conditions of this study). The data suggest that changes to the airway wall shape or dimensions, or increased airway secretions, may exert a significant effect on airway flow. Such factors could influence responses in both central and peripheral airways, with small airways being more vulnerable because critical narrowing in response to muscle contraction is reached more quickly.

Acknowledgements: The authors thank G. Yates P. Oates and A. Light for contributions. T. Nguyen is thanked for technical assistance.

\section{References}

1. Moreno RH, Hogg JC, Pare PD. Mechanics of airway narrowing. Am Rev Respir Dis 1986; 133: 1171-1180.

2. Wiggs BR, Moreno R, Hogg JC, Hilliam C, Pare PD. A model of the mechanics of airway narrowing. $J$ Appl Physiol 1990; 69: 849-860.

3. James AL, Pare PD, Hogg JC. The mechanics of airway narrowing in asthma. Am Rev Respir Dis 1989; 139: 242246.

4. Lambert RK. Role of bronchial basement membrane in airway collapse. J Appl Physiol 1991; 71: 666-673.

5. Yager D, Butler JP, Bastacky J, Israel E. Amplification of airway constriction due to liquid filling of airway interstices. J Appl Physiol 1989; 66: 2873-2884.

6. Okazawa M, Bai TR,Wiggs BR, Pare PD. Airway smooth muscle shortening in excised canine lung lobes. $J$ Appl Physiol 1993; 74: 1613-1621.

7. Opazo-Saez A, Du T, Wang NS, Martin JG. Methacholine-induced bronchoconstriction and airway smooth muscle in the guinea pig. J Appl Physiol 1996; 80: 437444.

8. Nagase T, Moretto A, Ludwig MS. Airway and tissue behavior during induced constriction in rats: intravenous $v s$. aerosol administration. J Appl Physiol 1994; 76: 830-838.

9. Eidelman DH, Lei M, Ghezzo RH. Morphometry of methacholine-induced bronchoconstriction in the rat. $J$ Appl Physiol 1993; 75: 1702-1710.

10. Dandurand RJ, Wang CG, Phillips NC, Eidelman DH. Responsiveness of individual airways to methacholine in adult rat lung explants. J Appl Physiol 1993; 75: 364-372.

11. Brown RH, Herold CJ, Hirshman CA, Zerhouni EA, Mitzner W. Individual airway constrictor response heterogeneity to histamine assessed by high-resolution computed tomography. J Appl Physiol 1993; 74: 2615-2620.

12. McNamara AE, Muller NL, Okazawa M, Arntorp J, Wiggs BR, Pare PD. Airway narrowing in excised canine lungs measured by high-resolution computed tomography. $J$ Appl Physiol 1992; 73: 307-316.

13. Shioya T, Solway J, Munoz NM, Mack M, Leff AR. Distribution of airway contractile responses within the major diameter bronchi during exogenous bronchoconstriction. Am Rev Respir Dis 1987; 135: 1105-1111.

14. Ludwig MS, Shore SA, Anderson K, Drazen JM. Temporal and regional variability of collateral resistance response to histamine. J Appl Physiol 1988; 64: 2142-2149.

15. Gray PR, Mitchell HW. Effect of diameter on force generation and responsiveness of bronchial segments and rings. Eur Respir J 1996; 9: 500-505.
16. Gunst SJ, Stropp JQ. Pressure-volume and length-stress relationships in canine bronchi in vitro. J Appl Physiol 1988; 64: 2522-2531.

17. Mitchell HW, Sparrow MP. Increased reponsiveness to cholinergic stimulation of small compared to large diameter cartilaginous bronchi. Eur Respir J 1994; 7: 298-305.

18. Sparrow MP, Mitchell HW. Modulation by the epithelium of the extent of bronchial narrowing produced by substances perfused through the lumen. $\mathrm{Br} J$ Pharmacol 1991; 103: 1160-1164.

19. McFawn PK, Sparrow MP, Mitchell HW. Measurement of flow through perfused bronchial segments: optimization of flow head resistance. Eur Respir J 1995; 8: 161-166.

20. Mitchell HW, Sparrow MP. Video-imaging of lumen narrowing; muscle shortening and flow responsiveness in isolated bronchial segments of the pig. Eur Respir $J$ 1994; 7: 1317-1325.

21. James AL, Hogg JC, Dunn LA, Pare PD. The use of the internal perimeter to compare airway size and to calculate smooth muscle shortening. Am J Respir Dis 1988; 138: 136-139.

22. Ding DJ, Martin JG, Macklem PT. Effects of lung volume on maximal methacholine induced bronchoconstriction. $J$ Appl Physiol 1987; 62: 1324-1330.

23. Gunst SJ, Warner DO, Wilson TA, Hyatt RE. Parenchymal interdependence and airway response to methacholine in excised dog lobes. J Appl Physiol 1988; 65: 2490-2497.

24. Tepper RS, Gunst SJ, Doerschuk CM, Shen X, Bray W. Effect of transpulmonary pressure on airway closure in immature and mature rabbits. J Appl Physiol 1995; 78: 505-512.

25. Sparrow MP, McFawn PK, Omari TI, Mitchell HW. Activation of smooth muscle in the airway wall, force production, and airway narrowing. Can J Physiol Pharmacol 1992; 70: 607-614.

26. Mitchell HW, Sparrow MP, Grayson PS. Structural properties of the bronchial wall determine the response of airway narrowing to external pressure. Am J Respir Crit Care Med 1994; 149: A585.

27. Bates JHT, Martin JG. A theoretical study of the effect of ASM orientation on bronchoconstriction. J Appl Physiol 1990; 69: 995-1001.

28. Murphy TM, Roy L, Phillips IJ, et al. Effect of maturation on topographic distribution of bronchoconstrictor responses in large diameter airway of young swine. Am Rev Respir Dis 1991; 143: 126-131.

29. Shioya T, Munoz NM, Leff AR. Effect of resting smooth muscle length on contractile response in resistance airways. J Appl Physiol 1987; 62: 711-717.

30. Brown RH, Georgakopoulos J, Mitzner W. Individual canine airways responsiveness to aerosol histamine and methacholine in vivo. Am J Respir Crit Care Med 1998; 157: 491-497.

31. Okazawa M, Vedal S, Verburgt L, Lambert RK, Pare PD. Determinants of airway smooth muscle shortening in excised canine lobes. J Appl Physiol 1995; 78: 608-614.

32. Brown RH, Mitzner W. Effect of lung inflation and airway muscle tone on airway diameter in vivo. J Appl Physiol 1996; 80: 1581-1588.

33. Mitchell HW, Gray PR. Uncoupling in the wall of the cartilaginous bronchus of the pig produced by smooth muscle contraction. Pulm Pharmacol 1996; 9: 29-34.

34. Stephens NL, Jiang He. Basic physiology of airway smooth muscle. In: Busse WW, Holgate ST, eds. Asthma and Rhinitis, Oxford, Blackwell Scientific Publications, 1995; Chap. 83, pp. 1087-1115.

35. Sasaki F, Saitoh Y, Verburgt L, Okazawa M. Airway wall dimensions during carbachol-induced bronchoconstriction in rabbits. J Appl Physiol 1996; 81: 1578-1583. 